\title{
Performance of Nanoparticles in the Electrical Behavior of DC Capacitor Films
}

\author{
Gian Carlo Montanari ${ }^{1,2}$, Paolo Seri ${ }^{1}$, Mikael Ritamäki ${ }^{3}$, Kari Lahti ${ }^{3}$, Ilkka Rytöluoto ${ }^{3}$ and Mika Paajanen ${ }^{4}$ \\ ${ }^{1}$ DEI, University of Bologna, Italy \\ ${ }^{2}$ Center for Electromechanics, University of Texas, USA \\ ${ }^{3}$ Tampere University of Technology, Electrical Energy Engineering, Tampere, Finland \\ ${ }^{4}$ VTT Technical Research Centre of Finland Ltd., Tampere, Finland
}

\begin{abstract}
In this work space charge accumulation characteristics of nanostructured PP-SiO2 films are investigated, providing preliminary results at the basis of the new materials formulations developed in the European project GRIDABLE, where novel MV and LV DC capacitor films having enhanced performance with respect to present polypropylene films are being developed. This paper shows that nanostructuration may be beneficial, especially at higher temperature, to improve material performance. Better space charge performance will help in optimizing the dielectric strength, design field and reliability of the insulation system for DC use.
\end{abstract}

\section{INTRODUCTION}

As the use of DC technology is increasing in the transfer of electricity over long distances or in relation to integration of the renewable energy sites into the main grid, there are specific challenges with the traditionally used insulating materials. Voltage source converter (VSC) based DC technology is the newest and 'smartest' way for connecting both renewable energy production and stationary energy storage units into $\mathrm{AC}$ grids. In addition, VSC is utilized to transmit and distribute electrical energy over long distances or from DC energy sources to users. VSC technology, together with energy storage, improves the power quality, transient and voltage stability, reduces losses and increases power oscillation damping. Furthermore, self-commutation of VSC enables the so called black start, i.e. starting of power injection into a nonenergized network, for example from stationary energy storage. This way VSC improves the overall reliability of the grid and VSC technology can also enable an increase in distributed energy source density, since otherwise power quality and stability issues normally limit the amount of distributed production in a network.

The technological and economic potential of voltage source converter based DC transmission technology can be further increased by introducing new advanced $\mathrm{PP} / \mathrm{SiO}_{2}$ materials into these systems. However, long-term performance of such new nanostructured material needs careful investigation.

Accumulation of space charge can be the main degradation process in DC insulations, since it can distort and enhance the Laplacian electric field distribution inside insulation, thus bring the maximum electric field amplitude to exceed the design field. This would shorten considerably life compared to the requested performance, considering, in particular, that electrical life line of insulation systems can be described by an inverse power law, [1], thus a small variation of electric field translated into large variation of life (e.g. $5 \%$ field increase can shorter life of one order of magnitude) [2], [3].
If voltage polarity inversions phenomena are also present, the accumulation of homocharge (that is, charge having the same sign as that of the near electrode) can bring to further abnormal field distribution, which can affect apparatus reliability and life. Any material which has to be used as DC insulation, therefore, has to be characterized to be space charge free at the operating field and temperature. Nanostructuration can help increasing field and/or temperature above which space charge accumulation becomes large enough to accelerate local degradation mechanisms [2]-[7].

\section{MATERIAL SPECIFICATIONS}

The new DC insulation technology characterized in this work had been developed in previous studies. It is a capacitorgrade isotactic polypropylene homopolymer matrix, with 4.5 wt- $\%$ of silica nanoparticles. An antioxidant package was added to prevent thermo-oxidative degradation of the PPmatrix during melt processing. An otherwise similar reference film was made without silica. The manufacturing details were reported in and the oriented films are the same studied in some papers, e.g. [8]. The thickness of the oriented films, Ref-BOPP and $\mathrm{SiO}_{2}-\mathrm{BOPP}$ are in the range of $15-18 \mu \mathrm{m}$. In addition to the oriented films, space charge in thick cast film specimens of similar films was studied. The cast films were from the reference and from two nanofilled PP grades with different screw speeds.

\section{SPACE CHARGE MEASUREMENTS}

Space charge measurements were carried out by the Pulsed Electro-Acoustic (PEA) method. In the PEA method, a specimen is subject to the superposition of a DC voltage U0 and a pulsed voltage $\mathrm{Up}(\mathrm{t})$. While the application of $\mathrm{DC}$ voltage is responsible for the formation of space charge in the material, pulses are necessary to generate a perturbation on the electric field acting on those charges. The resulting electric force pulse on charges causes them to accelerate and interact with the surrounding dielectric material. Such interaction initiates two acoustic pressure waves travelling in opposite directions and parallel to the electric field orientation, through the thickness of the sample. The wave moving towards the ground electrode is transferred through the whole thickness of the electrode itself, finally being acquired by a piezoelectric sensor and translated into a voltage signal. In this method, several factors limit the spatial resolution achievable. Pulse width, piezoelectric thickness and the bandwidth of the electronics utilized for signal amplification are the main limiting factors. 
In this work two PEA systems were used, one for thick cast film samples and another for thin oriented films. Both systems utilize $10 \mathrm{~ns}$ pulses with an intensity of $300 \mathrm{~V}$ for thin films and $580 \mathrm{~V}$ for cast films. The acoustic signal is collected by a 9 $\mu \mathrm{m}$ thick PVDF piezoelectric and amplified by an amplifier cascade. The thin film system amplifier has a $-3 \mathrm{~dB}$ bandwidth of $200 \mathrm{MHz}$. These features resulted in a spatial resolution of few tens of micrometers. This is comparable to the thickness of the specimens, therefore information on exact charge density vs. distance is partially lost, but a clear measurement of accumulated space charge during and after a period of polarization can be derived. Other interesting quantities can be extracted from space charge trends in depolarization, that is, apparent charge mobility and trap depth distribution [9]. Those are fundamental parameters for the characterization of insulating materials for DC applications, because the presence of space charge in the insulation can modify substantially the electric field distribution and, hence, the insulation life [1]-[7].

Calibration of the system is made after $10 \mathrm{~s}$ from polarization of the sample, through the following equations:

$$
\begin{gathered}
E(z)=\frac{\int_{0}^{z} \rho(z) d z}{\varepsilon} \\
\rho=\varepsilon E_{D C}
\end{gathered}
$$

Where $E(z)$ and $\rho(z)$ are the electric field and the charge density in the thickness of the specimen, $\varepsilon$ is the permittivity of the specimens tested and $E_{\mathrm{DC}}$ and $\rho$ are the theoretical electric field and surface charge density when no space charge is accumulated in the sample. Stored charge density at a specific field and depolarization time, $q(E, t)$, derived from the space charge profile measurements, as follows:

$$
q(E, t)=\frac{1}{L} \int_{0}^{L}\left|q_{p}(E, x, t) d x\right|
$$

where 0 and $L$ denote the electrodes positions and $q_{p}(E, x, t)$ is the space charge profile for a given poling field $\mathrm{E}$.

Trap controlled apparent mobility can be roughly evaluated from the $q$ vs $t$ characteristic as [9]:

$$
\mu(t)=\frac{\varepsilon}{q^{2}(t)} \frac{d q(t)}{d t}
$$

where $q(t)$ is the charge density that can be calculated at any depolarization time and $d q(t) / d t$ is the slope of the depolarization curve at time $t$. Once the apparent trapcontrolled mobility is known, the trap depth distribution, $\Delta U$, could be estimated, as described in [9], which provides the following equation:

$$
\Delta U(t)=K T \ln \left(\mu(t) \frac{h}{e R^{2}}\right)
$$

where $K$ is Boltzman constant, $T$ is temperature, $R$ is the mean distance between localized states, $e$ is the electron charge and $h$ is the Planck's constant. Therefore, the values of the trap depth can be determined from the values previously calculated for $\mu(t)$.

Complex permittivity measurements within our research group suggest that there is negligible water absorption from the atmosphere in these materials, therefore space charge measurements were conducted on cast and oriented films as received. Polarization time (when DC voltage is applied) was $1800 \mathrm{~s}$, followed by 600 seconds of depolarization (when DC voltage is absent). A mechanical switch was used to short the sample in the beginning of the depolarization. Tests were done at room temperature, $40^{\circ} \mathrm{C}$ and $60^{\circ} \mathrm{C}$, in a controlled environment, with fields of 200,300 and $400 \mathrm{kV} / \mathrm{mm}$, to obtain indications about the threshold conditions for space charge accumulation, as well as accumulation rate as function of field. The test voltages were chosen by assuming a uniform thickness of $16 \mu \mathrm{m}$; the thicknesses were measured afterwards the actual fields were determined. The chosen stresses are comparable to operating conditions of DC metallized-film capacitors.

\section{SPACE CHARGE ACCUMULATION PERFORMANCE}

Figures 1 and 2 show the signal amplitude from thin film PEA measurements at $40^{\circ} \mathrm{C}$, in poling fields from 200 to 400 $\mathrm{kV} / \mathrm{mm}$. The PEA signal amplitude originates from the superposition of Laplacian field (voltage at electrodes) and image field (space charge), hence if the measurement the voltage is kept constant any change in the amplitude is caused by space charge density variation. Very little differences were noticed for filled and non-filled materials both during polarization and depolarization periods (Fig. 1 A, B). During polarization (Fig.1), after an initial transient of less than 5 second, PEA signal amplitude remains stable. This is a clear indication that little to no charge is stored in the sample during polarization. When depolarization is started (Fig.3), PEA signals for filled and non-filled materials drop to similarly low asymptotic levels, with comparable time dynamics. This suggests that those two materials are characterized by comparable levels of traps depth distributions. Similar performance was also observed at room temperature. In order to enhance the relevance of this investigation and to approach conditions more similar to real working conditions for such materials, tests were run at the higher temperature of $60^{\circ} \mathrm{C}$. The effect of introducing a $4.5 \%$-wt of nanofiller under poling fields of 200 to $400 \mathrm{kV} / \mathrm{mm}$ can be now noticed in Figures 2 and 4 . During polarization at $60^{\circ} \mathrm{C}$ the PEA signal amplitude of non-filled material increases, while it stays flat in the filled material. This is an interesting difference, since it is a clear suggestion that while the non-filled material has the tendency to increase the amount of stored charge over time (Fig.4A), this is not true for the filled one (Fig.4B), showing a flat trend of PEA signal during the polarization period. 

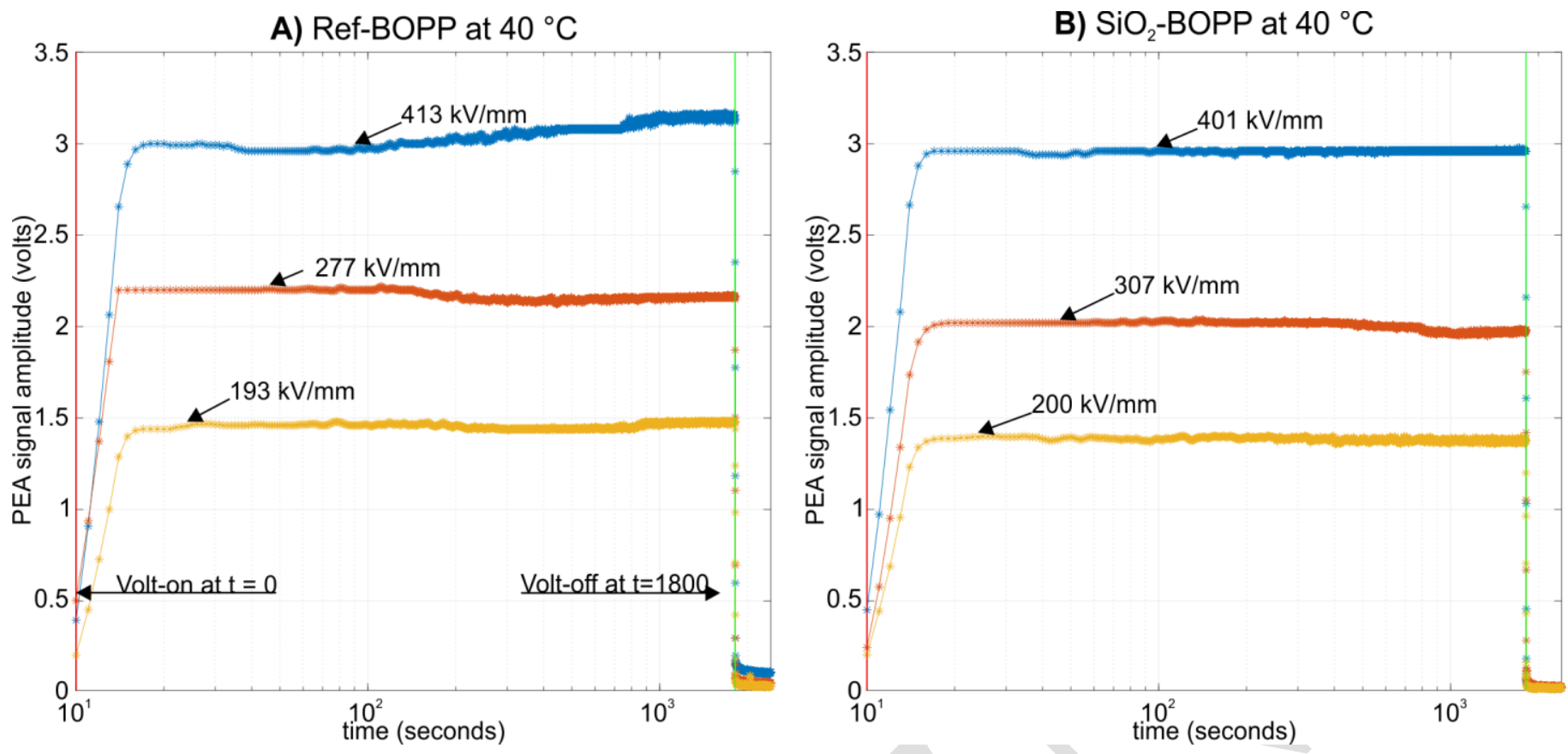

Fig. 2. PEA signal trends at $40^{\circ} \mathrm{C}$ for non-filled (A) and filled (B) materials. Depolarization period starts in correspondence with the green line.
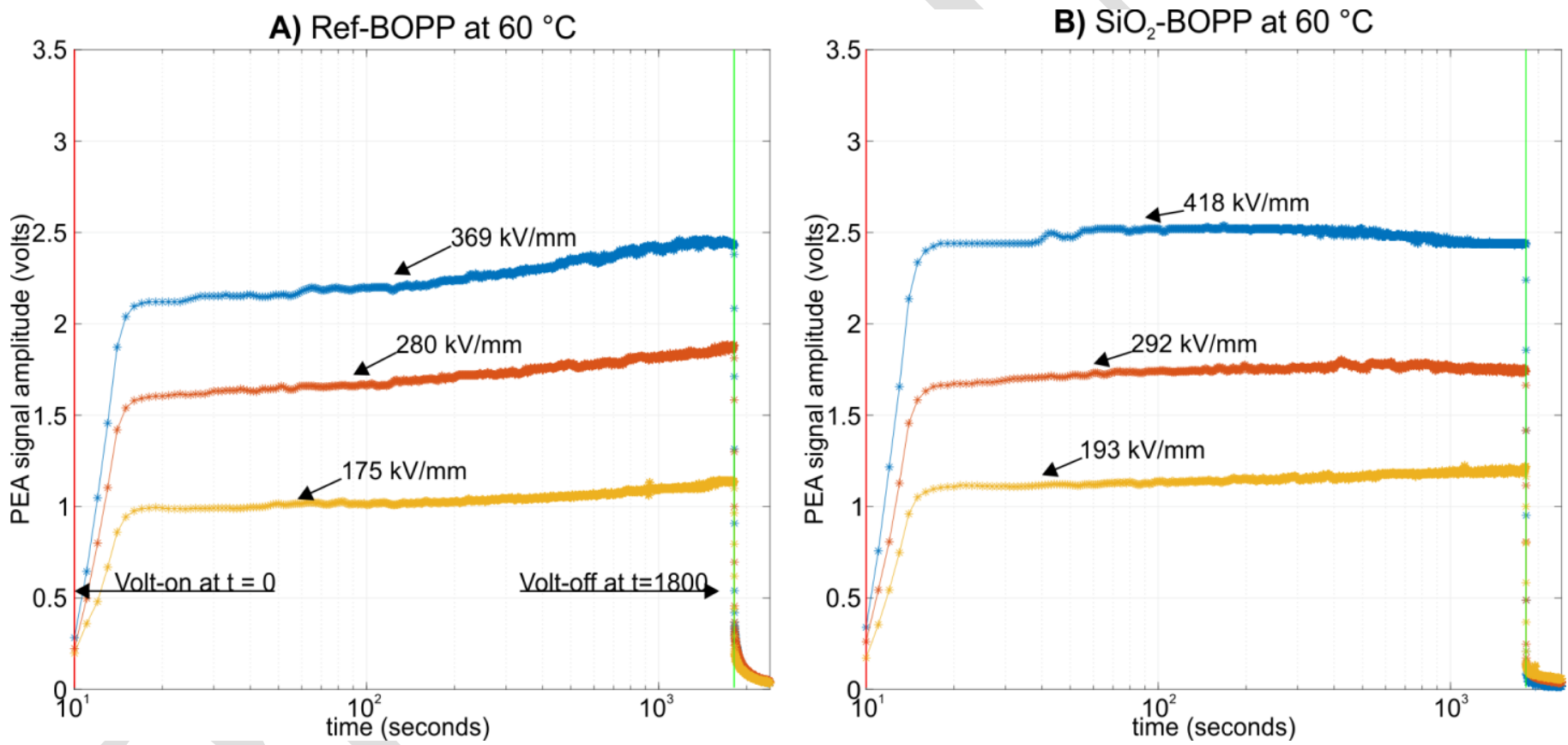

Fig. 1. PEA signal trends at $60^{\circ} \mathrm{C}$ for non-filled (A) and filled (B) materials. Depolarization period starts in correspondence with the green line.

A second interesting development highlighted by the investigation done at $60^{\circ} \mathrm{C}$ is the increase of charge dissipation dynamics of bulk space charge when the nanofiller is introduced. In fact, Figure 4 displays a clear modification of the PEA signal behavior over time during the depolarization periods, showing how filled materials have faster dissipation rate of charge over time, when no poling field is applied. To study whether similar performance improvement is seen also in cast films, PEA measurements were done at $60{ }^{\circ} \mathrm{C}$, poling the samples for 10000 seconds at $30 \mathrm{kV} / \mathrm{mm}$. The results, as summarized in Figure 5, were similar to those obtained for oriented films, with filled-PP displaying less charge accumulation. The non-filled PP accumulated more space charge, and the trapped charge decays more slowly than for nanofilled PP. This observation is especially interesting since the thin films were measured at 10 times or higher fields, meaning that space charge measurements have the potential to offer a viable way to "screen" a selection of thin films compositions by a preliminary investigation on thicker cast films, before orientation, despite the technical limits preventing testing at fields close to the typical design conditions for those materials. Calculations for the apparent trap depth distribution are supporting the already discussed indications given by the depolarization trends. Nanofilled materials show higher mobilities and lower trap depth distributions than the reference. This is an important characteristic for HVDC materials, since higher charge mobility or lower trap depth distribution contributes to a quicker stored charge removal, therefore a lower field enhancement in the case of polarity inversion and quicker restoration of the material natural charge neutrality. 


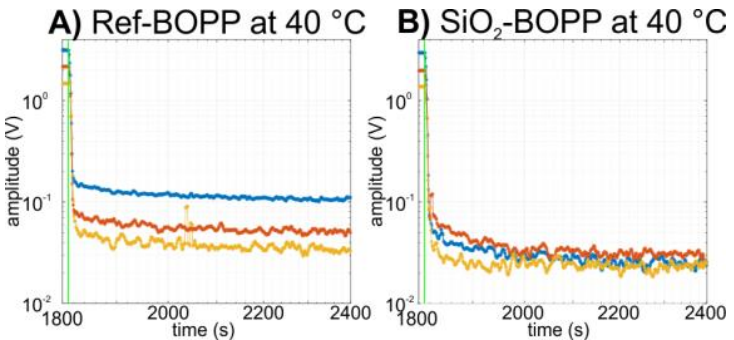

Fig. 3 PEA signal trends during depolarization at $40^{\circ} \mathrm{C}$ for non-filled and filled materials.

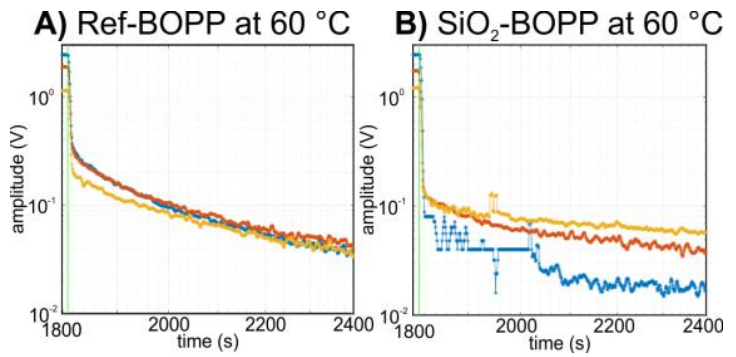

Fig. 4 PEA signal trends during depolarization at $60^{\circ} \mathrm{C}$ for non-filled and filled materials.

\section{CONCLUSIONS}

The addition of $\mathrm{SiO}_{2}$ nanoparticles to a bulk of PP has revealed interesting modifications of electrical properties that may affect positively film capacitors design and performance on short term and long term basis. This work shows that the introduction of nanofillers reduce the amount of accumulated charge at medium-high fields. This improvement was seen both in oriented and non-oriented cast films. On the other hand, charge depletion rate increases significantly from base (pure) to filled material, so that charge is released considerably faster for nanocomposites than for base (unfilled) materials. This behavior became prominent with increasing temperature. These results suggest a modification of trap density and trap depth distribution, confirmed by the depolarization space charge characteristics. Trapped charge is released, in fact, (as it could be expected due to the large interaction between nanofiller surface and host material) more rapidly by the nanofilled materials, and the residual charge after long depolarization times is smaller in nanofilled materials than in the base PP. As a consequence, trap-controlled apparent mobility increases significantly for nanofilled materials. Trap depth distribution seems to involve larger density of shallow traps compared to unfilled material (see Figure 5). The reduction of accumulated space charge would indicate that most of these carriers, as well as electrode-injected carriers, crossing insulation through shallow traps are more easily extracted at the electrodes. The results shown in this work set encouraging preliminary results for the GRIDABLE project: PEA measurements on thin and thick samples proved to deliver consistent results, suggesting a fast and viable way to preliminarily select a variety of materials at the early stages of new thin film materials development. Further, long term, investigations are needed to understand the contribution of nanofillers (both their nature and quantity) to the modification of electrical properties.

\section{ACKNOWLEDGMENT}

GRIDABLE is an industrially-driven consortium consisting of 3 industrial partners (GE Grid Solutions, Nexans and

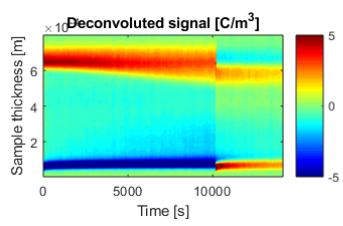

Injected charge $[\mathrm{C} / \mathrm{m} 3]$
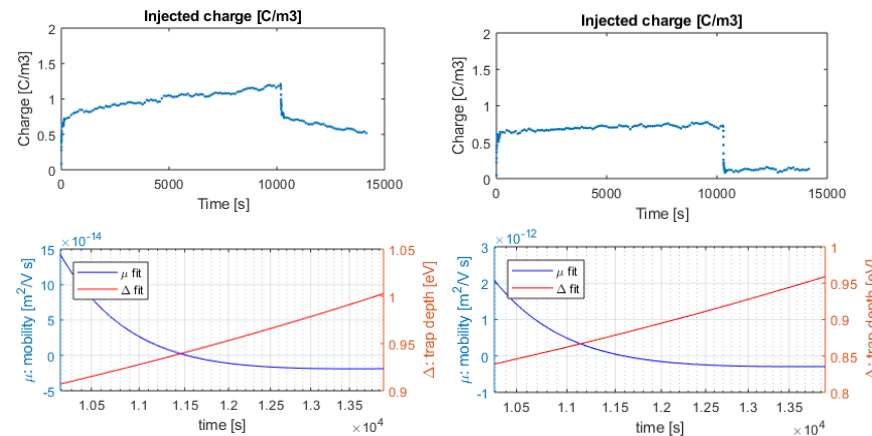

Fig. 5- Apparent charge mobility and trap depth at $60^{\circ} \mathrm{C}$ for non-filled (A) and filled (B) materials. Results from thick cast film samples.

Terichem), 1 consulting company (InnoEXC), 1 research institute (VTT) and 3 universities (University of Bologna, Tampere University of Technology and University of Twente) This project has received funding from the European Union's Horizon 2020 research and innovation programme under grant agreement No 720858

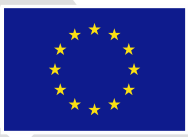

\section{REFERENCES}

[1] G.C. Montanari, G. Mazzanti, L. Simoni, "Progress in electrothermal life modeling of electrical insulation during the last decades", IEEE Trans. on Dielectrics and Electrical Insulation, Vol. 9, n. 5, pp. 730-745, October 2002.

[2] G. C. Montanari and G. Pattini, "Thermal Endurance Evaluation of Insulating Materials: A Theoretical and Experimental Analysis," in IEEE Transactions on Electrical Insulation, vol. EI-21, no. 1, pp. 69-77, Feb. 1986

[3] Cavallini, D. Fabiani, G. Mazzanti, and G. C. Montanari, "Life model based on space-charge quantities for HVDC polymeric cables subjected to voltage-polarity inversions," IEEE Trans. Dielectr. Electr. Insul., vol. 9, no. 4, pp. 514-523, Aug. 2002.

[4] G. Montanari, "Bringing an insulation to failure: the role of space charge," IEEE Trans. Dielectr. Electr. Insul., vol. 18, no. 2, pp. 339-364, Apr. 2011.

[5] G. C. Montanari, "The electrical degradation threshold of polyethylene investigated by space charge and conduction current measurements," IEEE Trans. Dielectr. Electr. Insul., vol. 7, no. 3, pp. 309-315, Jun. 2000 .

[6] G. Mazzanti, G. C. Montanari, and L. A. Dissado, "Electrical aging and life models: the role of space charge," IEEE Trans. Dielectr. Electr. Insul., vol. 12, no. 5, pp. 876-890, Oct. 2005.

[7] G. C. Montanari, "Relation between space charge and polymeric insulation ageing: cause and effect," IEE Proc. - Sci. Meas. Technol., vol. 150 , no. 2 , pp. 53-57, Mar. 2003.

[8] M. Ritamäki, I. Rytöluoto, K. Lahti, and M. Karttunen, "Effects of thermal aging on the characteristic breakdown behavior of Nano-SiO2BOPP and BOPP films," in 2015 IEEE 11th International Conference on the Properties and Applications of Dielectric Materials (ICPADM), 2015, pp. 400-403.

[9] G. Mazzanti, G. C. Montanari, and J. M. Alison, "A space-charge based method for the estimation of apparent mobility and trap depth as markers for insulation degradation-theoretical basis and experimental validation," IEEE Trans. Dielectr. Electr. Insul., vol. 10, no. 2, pp. 187-197, Apr. 2003. 